\title{
Proceedings of the 37th International Symposium on Automation and Robotics in Construction (ISARC 2020)
}

\author{
From Demonstration to Practical Use \\ - To New Stage of Construction Robot -
}

Kitakyushu, Japan, October 27-28, 2020

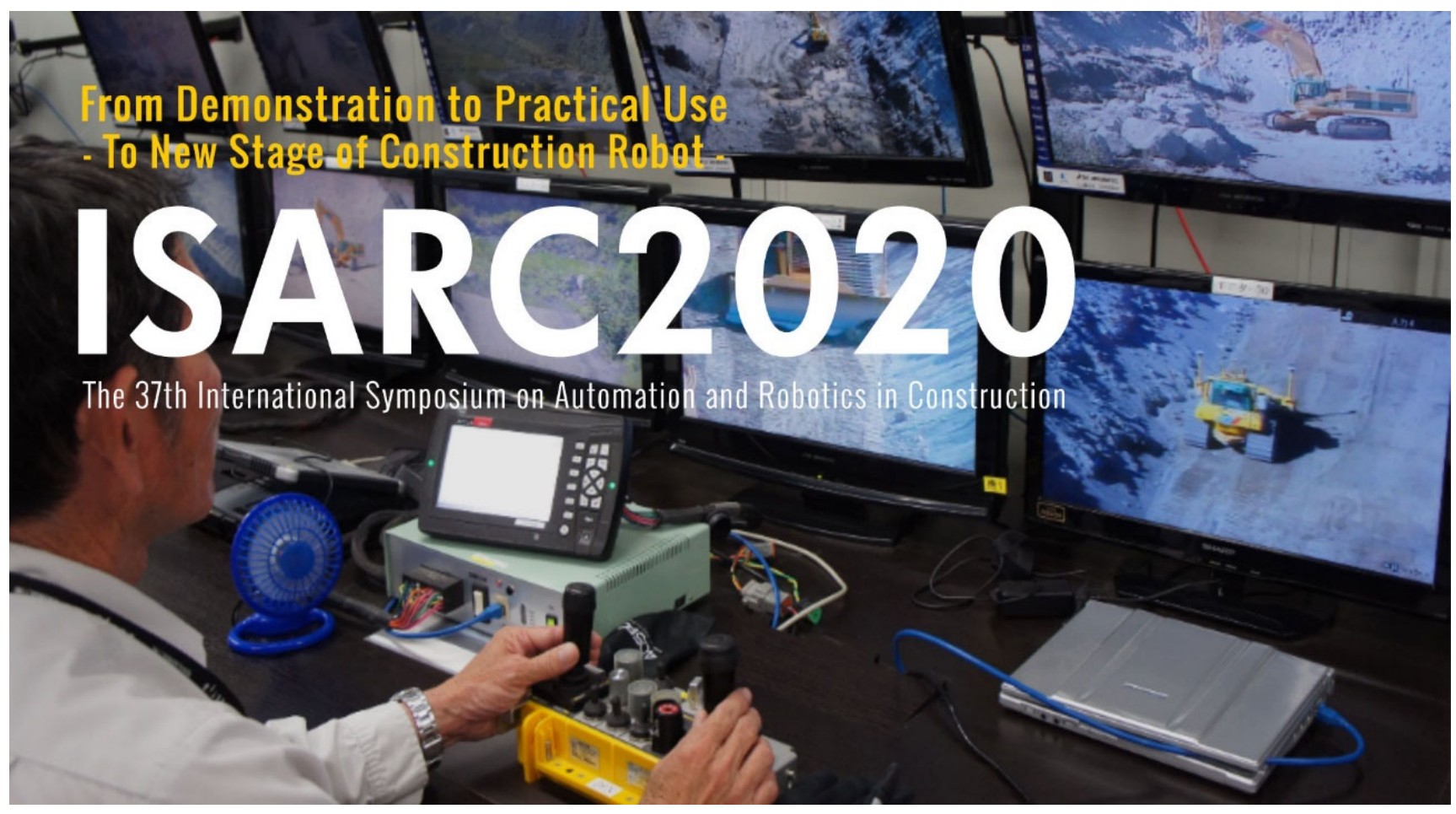

$37^{\text {th }}$ ISARC 2020 Online

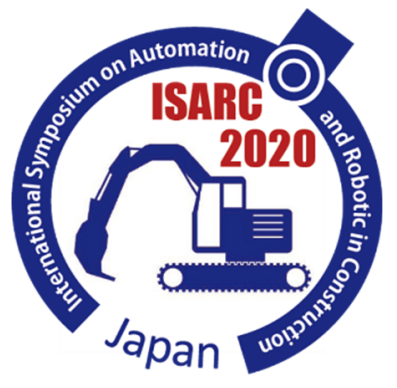

International Association for Automation and Robotics in Construction

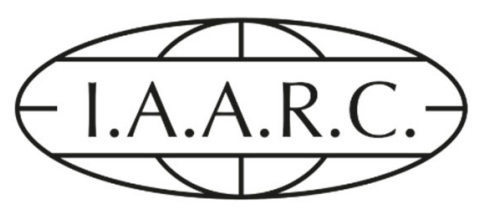

Knowledge and Competence 
Kazuyoshi Tateyama

Ritsumeikan University

Japan

Kazuo Ishii

Kyushu Institute of Technology

Japan

Fumihiro Inoue

Shonan Institute of Technology

Japan

ISBN 978-952-94-3634-7

1. Edition 2020

All rights reserved

(C) 2020 International Association on Automation and Robotics in Construction

The work including all its parts is protected by copyright. Any use outside the narrow limits of copyright law without the consent of the individual authors is inadmissible and punishable. This applies in particular to reproductions, translations, microfilming and saving and processing in electronic systems.

The reproduction of common names, trade names, trade names etc. in this work does not justify the assumption that such names are to be regarded as free within the meaning of the trademark and trademark protection legislation and can therefore be used by everyone, even without special identification.

Cover design: Masaharu Moteki 


\section{Preface}

The 1st International Symposium on Automation and Construction (ISARC) was held at Carnegie Mellon University in Pittsburgh, Pennsylvania, USA in 1984. Since then, ISARC has been held all over the world every year to exchange information about the development and practical use of construction robot technology among industry experts, academic researchers and individuals with novel ideas for all fields of construction, civil and building engineering, machine automation, robotics applications to construction, information technologies, planning, logistics, etc.

ISARC has been held in Japan four times so far, but it has not been held here since 2006, because the momentum for the development of construction robots declined rapidly with the economic downturn around 2000 . We were very pleased to learn that we would be able to hold the 37th ISARC in Japan in 2020 after 14 years thanks to the recent increase of momentum in the development of construction robots.

For the symposium, we had planned and prepared to organize not only research presentations, but also key note lectures, technical exhibitions and technical visits related to construction robots in use on the island of Kyushu, in the western part of Japan. Last December, we started a call for papers, and more than 390 abstracts were received from 33 countries.

Unfortunately, the infectious disease caused by COVID-19, which started at the end of last year, quickly spread throughout the world, and many people are still suffering from its effects. We would like to express our heartfelt sympathy to all the people who are in a severe situation, including those who have lost loved ones and/or have been infected by the disease.

There is still no clear end in sight to COVID-19. For this reason, in May of this year, we decided to hold the symposium online and immediately started the preparations for an online symposium. However, we had neither the experience nor the know-how to organize an online international symposium. Therefore, our original plan was not necessarily a very productive one. Under these circumstances, we received tremendous support from the IAARC Board members and were able to hold the online symposium successfully. We would like to extend our sincere gratitude to them for their kindness and great cooperation.

Although the number of submitted papers decreased, due to changes related to an online symposium, we still received 221 full papers from 23 countries. We believe that this symposium was very fruitful in terms of cross-national technical exchange among all the participants.

Finally, I would like to express our deepest gratitude to the members of the Japanese local committee for all the work they did with us in planning and preparing for this symposium. I believe that the efforts of all the people involved in this symposium will greatly contribute to the further evolution of construction robots.

Kazuyoshi Tateyama

Chair, 37th ISARC

Professor, Ritsumeikan University, Japan 


\section{Introduction}

This publication is the Proceedings of the 37th International Symposium on Automation and Robotics in Construction (ISARC). The symposium was held online during 27-28 October 2020. The Proceedings include an illustrated review of the program, the names of organizations and persons who contributed to the technical program, and the 221 technical papers from 23 countries authored for this international meeting.

The manuscripts were presented during 57 sessions on 3 tracks, among them: automation and robotics, building information modeling (BIM), inspection and monitoring, artificial intelligence and machine learning, construction management, safety and health, data sensing and analysis, mixed Realities (AR/VR), control technology, education, environmental sensing and modeling, human sensing and monitoring, IT supported system, database, big data, lean, logistics, prefabrication, modularization, leaning/Al/recognition, human-computer interaction, measurement, modeling and management, new application field of construction robots and machines, risk management, robot and interface design, and robot construction.

Please note: All ISARC proceedings since 1984 are available at no cost at http://www.iaarc.org.

We are very grateful for the support of so many. Thank you!

Prof. Kazuyoshi Tateyama, Ritsumeikan University, Japan (Chair)

Prof. Kazuo Ishii, Kyushu Institute of Technology, Japan (Co-Chair)

Prof. Fumihiro Inoue, Shonan Institute of Technology, Japan (Co-Chair) 


\section{Acknowledgements}

Symposium sponsors:

Symposium co-sponsors:

Symposium cooperation:

Symposium support:
Symposium host:

The International Association for Automation and Robotics in Construction Japanese Council for Construction Robot Research Council for Construction Robot Research The International Association for Automation and Robotics in Construction

$\begin{array}{ll}\text { Symposium co-sponsors: } & \text { Advanced Construction Technology Center } \\ & \text { Architectural Institute of Japan } \\ & \text { Japan Robot Association } \\ & \text { Japan Construction Machinery and Construction Association } \\ & \text { Japan Society of Civil Engineers } \\ & \text { City of Kitakyushu } \\ & \text { The Robotics Society of Japan } \\ \text { Symposium cooperation: } & \text { The Institute of Electrical Engineers of Japan } \\ & \text { Japan Association of Surveyors } \\ & \text { Japan Association for the Unmanned Construction } \\ & \text { Japan Federation of Construction Contractors } \\ & \text { The Japanese Geotechnical Society } \\ & \text { The Japan Society of Mechanical Engineers } \\ & \text { The Japan Society for Precision Engineering } \\ & \text { Kitakyushu Convention \& Visitors Association } \\ & \text { The Society of Instrument and Control Engineers } \\ & \text { Sabo \& Landslide Technical Center } \\ & \text { New Unmanned Construction Technology Research } \\ & \text { Association } \\ & \text { Ritsumeikan University } \\ \text { The Kajima Foundation } & \text { Rent All Scholarship Foundation } \\ \text { Ministry of Land, Infrastructure, Transport and Tourism }\end{array}$
Japan Robot Association Japan Construction Machinery and Construction Association City of Kitakyushu

The Institute of Electrical Engineers of Japan Japan Association of Surveyors Japan Association for the Unmanned Construction Japan Federation of Construction Contractors The Japanese Geotechnical Society The Japan Society of Mechanical Engineers The Japan Society for Precision Engineering Kitakyushu Convention \& Visitors Association Sabo \& Landslide Technical Center New Unmanned Construction Technology Research Association

Ritsumeikan University The Kajima Foundation Ministry of Land, Infrastructure, Transport and Tourism 


\section{Local Organizing Committee}

Chair

Co-Chair

Co-Chair
Kazuyoshi Tateyama, Ritsumeikan University, Japan

Fumihiro Inoue, Shonan Institute of Technology, Japan

Kazuo Ishii, Kyushu Institute of Technology, Japan

\section{Program committee}

Chair

Hisashi Osumi, Chuo University, Japan

Co-Chair

Kazuo Kikawada, Hazama Ando Corporation, Japan

Co-Chair

Fumihiro Inoue, Shonan Institute of Technology, Japan

Co-Chair

Mitsuo Kamesaki, Waseda University, Japan

Award committee

Chair

Miho Makatayama, Building Research Institute, Japan

\section{Publication committee}

Chair

Masamitsu Kurisu, Osaka University, Japan

\section{Web-management}

Chair

Takaaki Yokoyama, Ritsumeikan University, Japan

International relations

Chair

Hiroshi Furuya, Obayashi Corporation, Japan

Finance

Chair

Hiroki Murakami, IHI Corporation, Japan

\section{Secretary-general}

Masaharu Moteki, Advanced Construction Technology Center, Japan 


\section{Technical Committee}

\begin{tabular}{|c|c|c|c|}
\hline Tatsuo Arai & The Univ. of Electro-Communications & Soonwook Kwon & Sungkyunkwan University \\
\hline Mantha Bharadwaj & New York Univ. - Abu Dhabi & Junbok Lee & Kyung Hee University \\
\hline Thomas Bock & Technical University Munich & Nan Li & Tsinghua University \\
\hline Frederic Bosche & University of Edinburgh & Miho Makatayama & Building Research Institute \\
\hline Ioannis Brilakis & University of Cambridge & Leonardo Messi & Polytechnic Univ. of Marche \\
\hline Alessandro Carbonari & Polytechnic University of Marche & Naoki Mori & Taisei Corporation \\
\hline Daniel Castro & Georgia Institute of Technology & Masaharu Moteki & Advanced Construction \\
\hline Soungho Chae & Kajima Corporation & & Technology Center \\
\hline Hung-Ming Chen & Ntl Taiwan Univ. Science \& Tech. & Hiroki Murakami & IHI Corporation \\
\hline Po-Han Chen & Ntl Taiwan University & Keiji Nagatani & The University of Tokyo \\
\hline Yong Cho & Georgia Institute of Technology & Satoshi Nakamura & Tokyu Construction \\
\hline Satoru Doi & Obayashi Corporation & Takahiro Nakamura & Kajima Corporation \\
\hline Chen Feng & New York University & Tadashi Narise & Maeda Corporation \\
\hline Yutaro Fukase & Shimizu Corporation & Yasutoshi Nomura & Ritsumeikan University \\
\hline Hiromitsu Fujii & Chiba Institute of Technology & Ken Ooi & Komatsu Ltd. \\
\hline Hiroshi Furuya & Obayashi Corporation & Makoto Oshio & Kajima Corporation \\
\hline Borja Garcia de Soto & New York University - Abu Dhabi & Hisashi Osumi & Chuo University \\
\hline Jozef Gasparik & Slovak University of Technology & Benny Raphael & I.I.T. Madras \\
\hline Quang Ha & University of Technology, Sydney & Saiedeh Razavi & McMaster University \\
\hline Daniel Hall & ETH Zurich & Kazuyuki Sano & Taisei Corporation \\
\hline Kouji Hamada & Obayashi Corporation & Anoop Sattineni & Auburn University \\
\hline Amin Hammad & Concordia University & Isaac Shabtai & Israel Institute of Technology \\
\hline Takeshi Hashimoto & Public Works Research Institute & Yasuyuki Shingu & Shimizu Corporation \\
\hline Shigeki Hiraoka & Topcon Corporation & Shinya Suzuki & Toda Corporation \\
\hline Daehie Hong & Korea University Seoul & Piotr Szynkarczyk & Industrial Research Institute \\
\hline Koji Ihara & Asunaro Aoki Construction Co., Ltd. & & for Autom. \& Measurement \\
\hline Ryusei Ikeda & East Nippon Expressway Co., Ltd. & Hiroki Takabayashi & aT ROBOTICS Inc. \\
\hline Fumihiro Inoue & Shonan Institute of Technology & Manabu Takeishi & Hazama Ando Corporation \\
\hline Genya Ishigami & Keio University & Toshinari Tanaka & Port \& Airport Research Inst. \\
\hline Kazuo Ishii & Kyushu Institute of Technology & Jochen Teizer & University of Bochum \\
\hline \multirow[t]{2}{*}{ Fumio Itoh } & Japan Construction Machinery and & Yelda Turkan & Oregon State University \\
\hline & Construction Association & Takao Ueno & Tokyu Construction \\
\hline Yonghoon Ji & Japan Advanced Inst. Science \& Tech. & Kazunori Umeda & Chuo University \\
\hline Vineet Kamat & The University of Michigan & Tomohiro Umetani & Konan University \\
\hline Mitsuhiro Kamezaki & Waseda University & Enrique Valero & University of Edinburgh \\
\hline Kazuito Kamiyama & Takenaka Corporation & Frans van Gassel & Eindhoven Univ. of Technology \\
\hline Kazuya Kikawada & Hazama Ando Corporation & Koshy Varghese & I.I.T. Madras \\
\hline Huoungkwan Kim & Yonsei University & Józef Wrona & Wajskova Technical Academia \\
\hline Shigeo Kitahara & Kumagai Gumi Co., Ltd. & Hiroshi Yamamoto & Komatsu Ltd. \\
\hline Taizo Kobayashi & Ritsumeikan University & Shinya Yamamoto & Shimizu Corporation \\
\hline Markus König & University of Bochum & Takaaki Yokoyama & Ritsumeikan University \\
\hline Masamitsu Kurisu & Osaka University & Zhenhua Zhu & Wisconsin University \\
\hline
\end{tabular}




\section{Program Schedule}

\begin{tabular}{|c|c|c|c|}
\hline JS & & Tuesday October 27 & Wednesday October 28 \\
\hline 08:00 & $13: 00$ & $\begin{array}{c}\text { Opening Ceremony } \\
\text { Tucker-Hasegawa } 2020 \text { Award Keynote Lecture } \\
\text { Academic Presentations }\end{array}$ & Academic Presentations \\
\hline $14: 00$ & $15: 00$ & Keynote2 & Keynote3 \\
\hline $15: 00$ & 19:00 & Academic Presentations & $\begin{array}{c}\text { Academic Presentations } \\
\text { Award and Closing Ceremony }\end{array}$ \\
\hline $20: 00$ & $23: 00$ & Academic Presentations & \\
\hline
\end{tabular}

*Japan Standard Time

\section{Keynote}

\section{Tucker-Hasegawa 2020 Award Keynote Lecture}

\section{Hyoungkwan KIM}

Professor at Yonsei University, Korea

\section{Smart Safety Assurance for Temporary Structures}

Temporary structures on construction sites has been the major cause of worker fatalities. According to a Korean statistics report, about 300 people are losing precious lives each year due to accidents involving temporary structures. A new research program was launched this year to develop a smart safety assurance system that recognizes, evaluates, and predicts accident risks that may occur during the installation, dismantling, and operation of temporary structures. It is a part of the smart construction initiative sponsored by the Korean Ministry of Land, Infrastructure, and Transport, and the Korea Agency for Infrastructure Technology Advancement. The program was designed for developing technologies such as deep learning-based hazard identification, augmented reality-based risk warning, and smart mobility for intelligent sensing of construction sites, with a total budget of 12.5 billion ( $\$ 10.5$ million) over six years. The program has a clear goal of reducing the number of accidents related to temporary structures by more than $25 \%$ through the creation of a new construction culture, safety-related policies, and safety-related industries.

\section{Speaker profile:}

Hyoungkwan KIM, Ph.D. is a Professor of the School of Civil and Environmental Engineering at Yonsei University, Korea. His areas of research include construction automation, infrastructure adaptation to climate change, and project finance. He is the principal investigator of a $\$ 10.5$ million research program titled "Smart Safety Assurance for Temporary Structures," which is a part of smart construction initiative sponsored by the Korean Ministry of Land, Infrastructure, and Transport, and the Korea Agency for Infrastructure Technology and Advancement. He serves as Vice- President for the International Association for Automation and Robotics in Construction (IAARC), and Associate Editor for Journal of Computing in Civil Engineering, American Society of Civil Engineers (ASCE). He also served as Secretary General for Association for Engineering Education in Southeast Asia and the Pacific (AEESEAP). He has received six excellent teaching awards and an excellent research award from Yonsei University. More information on Prof. Kim can be found at: http://aim.yonsei.ac.kr. 


\section{Keynote 2}

\section{Naoki SATO}

Director of the Space Exploration System Technology Unit, The Japan Aerospace Exploration Agency (JAXA), JAXA Space Exploration Center (JSEC), Japan

\section{International Space Exploration and Japanese Lunar Activities}

JAXA is engaged in international collaborations to tackle the challenge of human and robotic exploration missions in and beyond low-Earth orbit (LEO). The current focus is exploration missions to the Moon and Mars, targeting future human activities. His presentation introduced Japan's current exploration activities and JAXA's future plans and studies beyond the Earth orbit with the context of international coordination. Especially for the lunar surface activities, the concept study of the lunar base construction, which JAXA had conducted with a group of construction-related companies across Japan, was introduced along with the technological development.

\section{Speaker profile:}

Naoki SATO graduated from the Aeronautics Engineering Department, Kyusyu University in 1986, and gained a master degree of applied engineering of Kyusyu University in 1988. In the same year, he entered the National Space Development Agency of Japan (predecessor of JAXA). From 1990 he had been involved in the International Space Station program for about 16 years. Afterwards, he has been working for the international space exploration program formulation. Since April 2018 he is the current ISECG chair and since July 2018 he was assigned as the Director of the Space Exploration System Technology Unit of JAXA Space Exploration Center (JSEC).

\section{Keynote 3}

\section{Yasushi NITTA}

Director for Construction Equipment and Safety Planning Office, Policy Bureau, Ministry of Land, Infrastructure, Transport and Tourism, Japan

\section{Initiatives for Robot Introduction in Japanese Public Works}

The Japanese society faces various social issues such as frequent occurrences of earthquakes, eruption of volcanoes, floods, landslides, etc., resulting in the deterioration of the infrastructure. Japan also sees a reduction of the working population in the construction industry. In his speech, he introduced initiatives for the social implementation of robots and information and communication technologies in the Japanese construction industry, including the Ministry of Land, Infrastructure, Transport and Tourism (MLIT).

\section{Speaker profile:}

After graduating from University of Tsukuba in 1994, Dr. Yasushi NITTA joined the Ministry of Land, Infrastructure, Transport and Tourism (MLIT). There he is widely engaged in policy planning, public works and R\&D in the various departments, such as MLIT Headquarters, Regional Development Bureau, National Road Office, National Research Institutes (PWRI, NILIM), Advanced Construction Technology Center (ACTEC). He is especially responsible for the planning and operation of on-site verification projects to promote the introduction of robots to the infrastructure department, development/deployment/budgeting/operation of disaster 
countermeasure machines, and nationwide deployment of machine construction (iConstruction) using 3D data. Dr. NITTA is also engaged in establishing technical standards for the purpose, demonstrating ultra-long-distance unmanned construction technology, and flood control as an international emergency relief team.

\section{Video list}

Construction robots in Japan

1. Sea Experiment on Tele-operation System of Underwater Excavator National Institute of Maritime, Port and Aviation Technology, Port and Airport Research Institute, Infrastructure Digital Transformation Engineering Department

2. Development of Heavy Carrier Robot for Shallow Water Area New Unmanned Construction Technology Research Association

3. Tunnel RemOS-WL Kanamoto Co., Ltd.

4. kana Robo - Robo-Construction System Kanamoto Co., Ltd.

5. kana Robo - Robo-Construction System 2 Kanamoto Co., Ltd.

6. ROBO CONSTRUCTION - DokaBOri Training Fujiken Co,Ltd.

7. "A4CSEL" at the Seisho Test and Practice Field KAJIMA CORPORATION

8. Pursuing "Zero Ground Subsidence" in Shield Tunneling TAC Corporation

9. Automatic Dam Concrete Placing System SHIMIZU CORPORATION

10. Automatic Tunnel Lining Concrete Placing System SHIMIZU CORPORATION

11. A robot that assists in plotting SHIMIZU CORPORATION

12. Development of IT construction system by Robot Public Works Research Institute

13. Demonstration of autonomous excavation, loading and unmanned bulldozer. (CEATEC2018) Komatsu Ltd., Office of CTO

14. Smart Construction Concept, Future image. (CEATEC2018) Komatsu Ltd., Office of CTO

15. BE A HERO, Future image Komatsu Ltd., Office of CTO

16. DEEP CRAWLER - Crawler type ROV WAKACHIKU CONSTRUCTION Co., Ltd.

17. What is dredging? - A job that protects the safety of the sea WAKACHIKU CONSTRUCTION Co., Ltd.

18. Robotic rubble-mound mechanized construction system PENTA-OCEAN CONSTRUCTION CO., LTD.

19. Rotation Control Device for Lifting Cargo WAKACHIKU CONSTRUCTION Co., Ltd.

20. Automatic operation system of the construction machine (Vibrating roller - Bulldozer) HAZAMA ANDO CORPORATION 


\section{Table of Contents}

Improving Construction Demonstrations by Integrating BIM, UAV, and VR

Kun-Chi Wang, Ren-Jie Gao, Sheng-Han Tung and Yuan-Hsiu Chou

Using Virtual Reality and Augmented Reality for Presale House Customer Change 8

Ben Amed Ouedraogo, Li-Chuan Lien, Unurjargal Dolgorsuren and Yan Ni Liu

Virtual Prototyping-Based Path Planning of Unmanned Aerial Vehicles for Building Exterior Inspection

Zhenjie Zheng, Mi Pan and Wei Pan

Near Real-Time Monitoring of Construction Progress: Integration of Extended Reality and Kinect V2 $\cdots$

Ahmed Khairadeen Ali, One Jae Lee and Chansik Park

VRGlare: A Virtual Reality Lighting Performance Simulator for real-time Three-Dimensional Glare

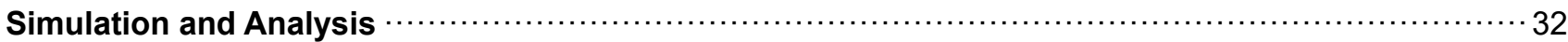

Kieran May, James Walsh, Ross Smith, Ning Gu and Bruce Thomas

Development of an Augmented Reality Fitness Index for Contractors 40

Hala Nassereddine, Wafik Lotfallah, Awad Hanna and Dharmaraj Veeramani

A Method to Produce \& Visualize Interactive Work Instructions for Modular Products within Onsite

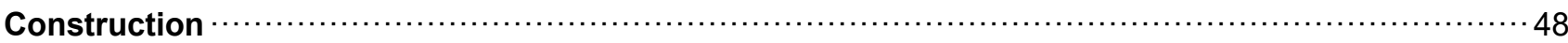

Raafat Hussamadin, Jani Mukkavaara and Gustav Jansson

A Framework for Augmented Reality Assisted Structural Embedment Inspection $\cdots \cdots \cdots \cdots \cdots \cdots \cdots \cdots \cdots \cdots \cdots \cdots \cdots \cdots$ Jeffrey Kim and Darren Olsen

Towards Circular Economy in Architecture by Means of Data-driven Design-to- Robotic-Production · 63 Ginevra Nazzari and Henriette Bier

Automated Pavement Marking Defects Detection 67

Andrea Leal Ruiz and Hani Alzraiee

An Assistive Interface of a Teleoperation System of an Excavator by Overlapping the Predicted Position of the Arm

Yuzuki Okawa, Masaru Ito, Ryota Sekizuka, Seiji Saiki, Yoichiro Yamazaki and Yuichi Kurita

Development of Rotary Snow Blower Vehicle Driving Support System using Quasi-Zenith Satellite on the Expressway in Japan .79

Katsuyoshi Abe, Atsushi Ichikawa, Toshiaki Itou and Keigo Kurihara

A Systematic Review of the Geographic and Chronological Distributions of 3D Concrete Printers from 1997 to 2020 .

Jihoon Chung, Ghang Lee and Jung-Hoon Kim 
Towards High-Quality Road Construction: Using Autonomous Tandem Rollers for Asphalt Compaction

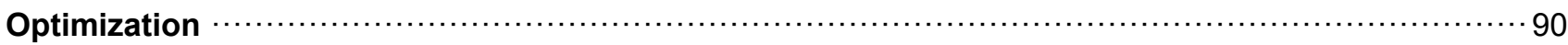

Jörg Husemann, Patrick Wolf, Axel Vierling, Karsten Berns and Peter Decker

Ontology-Based Decoding of Risks Encoded in the Prescriptive Requirements in Bridge Design Codes

Fahad UI Hassan and Tuyen Le

Current Status of Unmanned Construction Technology Developed using a Test Field System ….... 105 Koji Ihara and Takeshi Tamura

Remote Control Demonstration of the Construction Machine Using 5G Mobile Communication System

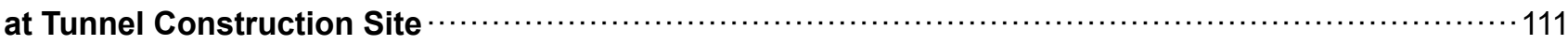

Ken Takai, Hiroaki Aoki, Yusuke Tajima and Michinobu Yoshida

Sea Experiment on Tele-operation System of Underwater Excavator

Tsukasa Kita, Taketsugu Hirabayashi, Atsushi Ueyama, Hiroshi Kinjo, Naoki Oshiro and Nobuyuki Kinjo

Automation and Operation Record of Large Overhead Crane for Segment Transportation 126

Yasushi Nishizaki, Koki Takahashi, and Takashi Fukui

Development of an Automated Angle Control System to Improve Safety and Productivity 134

Tsuyoshi Fukuda, Takumi Arai, Kousuke Kakimi and Keishi Matsumoto

Automated Detection for Road Marking Quality, using Visual Based Machine Learning 139

Firas Habbal, Fawaz Habbal, Abdualla Alnuaimi, Shafia Alkheyaili and Ammar Safi

A Construction Progress On-site Monitoring and Presentation System Based on The Integration of Augmented Reality and BIM ....

Sheng-Kai Wang and Hung-Ming Chen

Rule-Based Generation of Assembly Sequences for Simulation in Large-Scale Plant Construction · 155 Jan Weber, Jana Stolipin, Ulrich Jessen, Markus König and Sigrid Wenzel

An analysis of 4D-BIM Construction Planning: Advantages, Risks and Challenges

Pedram Farnood Ahmadi and Mehrdad Arashpour

Ontology-based Product Configuration for Modular Buildings Jianpeng Cao and Daniel Hall

On construction-specific Product Structure Design and Development: the BIM Enhancement Approach

Solmaz Mansoori, Harri Haapasalo and Janne Härkönen

Parametric Structural Design for automated Multi-Objective Optimization of Flexible Industrial Buildings Julia Reisinger, Maximilian Knoll and Iva Kovacic 
Hashim Raza Bokhari, Doyeop Lee, Numan Khan and Chansik Park

Status of 4D BIM Implementation in Indian Construction

V. Paul C. Charlesraj and Talapaneni Dinesh

An Information Quality Assessment Framework for Developing Building Information Models

Liji Chen and Justin K. W. Yeoh

BIM Based Information Delivery Controlling System

Brian Klusmann, Zhiwei Meng, Noemi Kremer, Anica Meins-Becker and Manfred Helmus

Development of an Open-source Scan+BIM Platform

Enrique Valero, Dibya D. Mohanty and Frederic Bosche

BuiltView: Integrating LiDAR and BIM for Real-Time Quality Control of Construction Projects 233

Rana Abbas, F. A. Westling, Christian Skinner, Monica Hanus-Smith, Andrew Harris and Nathan Kirchner

Single Shared Model Approach for Building Information Modelling 240

Simo Ruokamo and Heikkila Rauno

Parametric or Non-parametric? Understanding the Inherent Trade-offs between Forms of Object Representation ..... 248

Christopher Rausch, Yinghui Zhao and Carl Haas

Development of a Twin Model for Real-time Detection of Fall Hazards 256

Leonardo Messi, Alessandra Corneli, Massimo Vaccarini and Alessandro Carbonari

Automatized Parametric Modeling to Enhance a data-based Maintenance Process for Infrastructure

Buildings 264

Robert Hartung, Robin Schönbach, Dominic Liepe and Katharina Klemt-Albert

Gamification and BIM Teaching the BIM Method through a Gamified, Collaborative Approach 272

Carla Pütz, Christian Heins, Manfred Helmus and Anica Meins-Becker

Opportunities and Challenges of Digital Twin Applications in Modular Integrated Construction $\cdots \cdots 278$ Mingcheng Xie and Wei Pan

Integrating Industry 4.0 Associated Technologies into Automated and Traditional Construction $\cdots \cdots 285$ Fabiano Correa

System Development of an Augmented Reality On-site BIM Viewer Based on the Integration of SLAM and BLE Indoor Positioning

Yu-Cheng Liu, Jhih-Rong Chen and Hung-Ming Chen 
A Web-Based Approach to Dynamically Assessing Space Conflicts by Integrating BIM and Graph

Database

Wei-Ting Chien and Shang-Hsien Hsieh

Deployment of a Standardized BIM Modeling Guideline for the Planning and Construction Industry 313 Gamze Hort, Daiki John Feller, Anica Meins-Becker and Manfred Helmus

Design for Digital Fabrication: an Industry needs Analysis of Collaboration Platforms and Integrated Management Processes.

Ming Shan Ng, Marcella M. Bonanomi, Daniel M. Hall and Jürgen Hackl

A BIM-Based Approach for Optimizing HVAC Design and Air Distribution System Layouts in Panelized

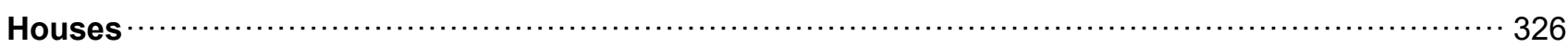

Pouya Baradaran-Noveiri, Mohammed Zaheeruddin and Sang Hyeok Han

Synthetic Data Generation for Indoor Scene Understanding Using BIM

Yeji Hong, Somin Park and Hyoungkwan Kim

Cyber-physical System for Diagnosing and Predicting Technical Condition of Servo-drives of Mechatronic Sliding Complex during Construction of High-rise Monolithic Buildings $\cdots \cdots \cdots \cdots \cdots \cdots \cdots \cdots \cdots \cdots$ Alexey Bulgakov, Thomas Bock and Tatiana Kruglova

Proposal for Automation System Diagram and Automation Levels for Earthmoving Machinery 347

Takeshi Hashimoto, Mitsuru Yamada, Genki Yamauchi, Yasushi Nitta and Shinichi Yuta

Applications of LiDAR for Productivity Improvement on Construction Projects: Case Studies from Active Sites

Fredrik Westling, Rana Abbas, Christian Skinner, Monica Hanus-Smith, Andrew Harris and Nathan Kirchner

Design and Construction of Shell-shaped Bench using a 3D Printer for Construction 362

Hajime Sakagami, Haruna Okawa, Masaya Nakamura, Takuya Anabuki, Yoshikazu Ishizeki and Tomoya Kaneko

Requirements for Safe Operation and Facility Maintenance of Construction Robots 369 Alexey Bulgakov, Thomas Bock, Jens Otto, Natalia Buzalo and Thomas Linner

Safety Concept and Architecture for Autonomous Haulage System in Mining 377 Hidefumi Ishimoto and Tomoyuki Hamada

On-site Autonomous Construction Robots: A review of Research Areas, Technologies, and Suggestions for Advancement 385

Xinghui Xu and Borja Garcia de Soto

Curtain Wall Installation for High-Rise Buildings: Critical Review of Current Automation Solutions and Opportunities

Brandon Johns, Mehrdad Arashpour and Elahe Abdi

Rationalization of Free-form Surface Construction Method using Wooden Formwork 401

Sei Hayashi and Tomoyuki Gondo 
Comparison of Shortest Path Finding Algorithms for Cable Networks on Industrial Construction

Projects

Fatima Alsakka, Salam Khalife, Maram Nomir, Yasser Mohamed and Rick Hermann

Constructability: The Prime Target in Value Engineering for Design Optimization

Arun Sekhar and Uma Maheswari

Blockchain based Framework for Verifying the Adequacy of Scaffolding Installation 425

Chanwoo Baek, Doyeop Lee and Chansik Park

Towards a Comprehensive Facade Inspection Process: An NLP based Analysis of Historical Facade Inspection Reports for Knowledge Discovery

Zhuoya Shi, Keundeok Park and Semiha Ergan

Scheduling Simulator by Ensemble Forecasting of Construction Duration 441

Shigeomi Nishigaki, Katsutoshi Saibara, Takashi Ootsuki and Hirokuni Morikawa

A Technology Platform for a Successful Implementation of Integrated Project Delivery for Medium Size Projects

Luke Psomas and Hani Alzraiee

Simulation-based Reinforcement Learning Approach towards Construction Machine Automation $\cdot 457$ Keita Matsumoto, Atsushi Yamaguchi, Takahiro Oka, Masahiro Yasumoto, Satoru Hara, Michitaka lida and Marek Teichmann

Optimization of Trajectories for Cable Robots on Automated Construction Sites 465

Roland Boumann, Patrik Lemmen, Robin Heidel and Tobias Bruckmann

A Robust Framework for Identifying Automated Construction Operations

Aparna Harichandran, Benny Raphael and Abhijit Mukherjee

Analysis of Excavation Methods for a Small-scale Mining Robot

Michael Berner and Nikolaus August Sifferlinger

Robotic Insertion of Timber Joints using Visual Detection of Fiducial Markers 491

Nicolas Rogeau, Victor Tiberghien, Pierre Latteur and Yves Weinand

Constraint Control of a Boom Crane System 499

Michele Ambrosino, Arnaud Dawans and Emanuele Garone

Optimal Travel Routes of On-road Vehicles Considering Sustainability

Nassim Mehrvarz, Zhilin Ye, Khalegh Barati and Xuesong Shen

Modeling and Control of 5-DoF Boom Crane

Michele Ambrosino, Marc Berneman, Gianluca Carbone, Rémi Crépin, Arnaud Dawans and Emanuele Garone

Automating Crane Lift Path through Integration of BIM and Path Finding Algorithm 522 Songbo Hu and Yihai Fang 
Hironobu Hatamoto, Kazuya Fujimoto, Tsubasa Asuma, Yoshito Takeshita, Tetsuo Amagai, Atsushi Furukawa and Shigeo Kitahara

Accuracy and Generality of Trained Models for Lift Planning Using Deep Reinforcement Learning Optimization of the Crane Hook Movement Between Two Points

Aoi Tarutani and Kosei Ishida

Reaching Difficulty Model of Swinging Operations of a Hydraulic Excavator Considering the First-Order Delay

Kazuyuki Matsumura, Masaru Ito, Chiaki Raima, Seiji Saiki, Yoichiro Yamazaki and Yuichi Kurita

Mechatronic Control System for Leveling of Bulldozer Blade 552 Alexey Bulgakov, Thomas Bock and Georgii Tokmakov

Multiple Tower Crane Selection methodology utilizing Genetic Algorithm 558 Preet Lodaya, Abhishek Raj Singh and Venkata Santosh Kumar Delhi

Fuzzy Controller Algorithm for Automated HVAC Control 566 Myungjin Chae, Kyubyung Kang, Dan D. Koo, Sukjoon Oh and Jae Youl Chun

A Probabilistic Motion Control Approach for Teleoperated Construction Machinery 571 Hyung Joo Lee and Sigrid Brell-Cokcan

Excavation Path Generation for Autonomous Excavator Considering Bulking Factor of Soil 578 Shinya Katsuma, Ryosuke Yajima, Shunsuke Hamasaki, Pang-Jo Chun, Keiji Nagatani, Genki Yamauchi, Takeshi Hashimoto, Atsushi Yamashita and Hajime Asama

Development of an Algorithm for Crane Sway Suppression 584

Yasuhiro Yamamoto, Chunnan Wu, Hisashi Osumi, Masayuki Yano and Yusuke Hara

Analysis of Energy Efficiency of a Backhoe during Digging Operation 589

Yusuke Sano, Chunnan Wu, Hisashi Osumi, Yuki Kawashima and Tomoaki Tsuda

Action Recognition of Construction Machinery from Simulated Training Data Using Video Filters $\cdots 595$ Jinhyeok Sim, Jun Younes Louhi Kasahara, Shota Chikushi, Hiroshi Yamakawa, Yusuke Tamura, Keiji Nagatani, Takumi Chiba, Shingo Yamamoto, Kazuhiro Chayama, Atsushi Yamashita and Hajime Asama

Real-time Aarly Warning of Clogging Risk in Slurry Shield Tunneling: A Self-updating Machine Learning Approach. 600

Qiang Wang, Xiongyao Xie and Yu Huang

Efficient Numerical Methods for Accurate Modeling of Soil Cutting Operations

Amin Haeri, Dominique Tremblay, Krzysztof Skonieczny, Daniel Holz and Marek Teichmann

loT-enabled Dependable Co-located Low-cost Sensing for Construction Site Monitoring 616

Huynh A. D. Nguyen, Lanh V. Nguyen and Quang P. Ha 
Threat Modeling in Construction: An Example of a 3D Concrete Printing System

Maahir Ur Rahman Mohamed Shibly and Borja Garcia de Soto

Measuring Adhesion Strength of Wall Tile to Concrete by Non-Contact Inspection Using

Electromagnetic Waves

Hussain Alsalem, Takayuki Tanaka, Takumi Honda, Satoru Doi and Shigeru Uchida

Construction Method of Super Flat Concrete Slab using High Precision Height Measurement 639

Yutaro Fukase, Ryosuke Saito, Yoshiaki Takemoto and Yoshiki Muramatsu

Method for Estimating Subgrade Reaction Modulus by Measuring Wheel-terrain Interactions 646 Yasushi Wada and Taizo Kobayashi

Report on the Measurement of the Form of SHOTCRETE GRID BEAM-FREE FRAME Using Point Cloud Data

Kojima Takayuki and Yori Nomoto

Single Camera Worker Detection, Tracking and Action Recognition in Construction Site 653 Hiroaki Ishioka, Xinshuo Weng, Yunze Man and Kris Kitani

MR-based Equipment Remote Control and 3D Digital Working Guidance for Field-oriented Maintenance 661

Jinwoo Song, Kyuhyup Lee, Minkyeong Jeong, Seojoon Lee and Soonwook Kwon

Use of Laser scanning, Remote Sensors \& Traffic Data Collection, Drones \& Mobile Application. MoEI Federal Highways network case study.

Khamis Al Sheyahri, Habiba Noor Aflatoon and Daniel Llort Mac Donald

Safety Monitoring of Construction Equipment based on Multi-sensor Technology

Ziqing Yang, Jian Yang and Enliu Yuan

Autonomous UAV flight using the Total Station Navigation System in Non-GNSS Environments 685

Akira Ishii, Takato Yasuno, Masazumi Amakata, Hiroaki Sugawara, Junichiro Fujii and Kohei Ozasa

Depth-Camera-Based In-line Evaluation of Surface Geometry and Material Classification For Robotic Spraying

Valens Frangez, David Salido-Monzú and Andreas Wieser

Combining Reality Capture and Augmented Reality to Visualise Subsurface Utilities in the Field $\cdots 703$ Lasse Hedegaard Hansen, Simon Swanström Wyke and Erik Kjems

Condition Prediction of Highway Assets Based on Spatial Proximity and Interrelations of Asset Classes: A Case Study

Arash Karimzadeh, Sepehr Sabeti, Hamed Tabkhib and Omidreza Shoghli

A Simulation Approach to Optimize Concrete Delivery using UAV Photogrammetry and Traffic Data $\cdots$

Robert Sprotte and Hani Alzraiee 
Quality Control for Concrete Steel Embed Plates using LiDAR and Point Cloud Mapping

Hani Alzraiee, Robert Sprotte and Andrea Leal Ruiz

Exploring Gerontechnology for Aging-Related Diseases in Design Education: An Interdisciplinary

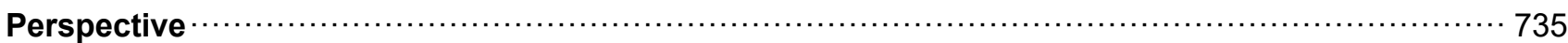

Rongbo Hu, Thomas Linner, Marc Schmaitzl, Jörg Güttler, Yuan Lu and Thomas Bock

Changing Paradigm: a Pedagogical Method of Robotic Tectonics into Architectural Curriculum $\cdots 743$ Xinyu Shi, Xue Fang, Zhoufan Chen, Tyson Keen Phillips and Hiroatsu Fukuda

Augmented Reality Sandboxes for Civil and Construction Engineering Education $\cdots \cdots \cdots \cdots \cdots \cdots \cdots \cdots \cdots \cdots \cdots \cdots$ Joseph Louis and Jennifer Lather

Education of Open Infra BIM based Automation and Robotics

Kolli Tanja and Heikkila Rauno

Automated Data Acquisition for Indoor Localization and Tracking of Materials Onsite 765 Hassan Bardareh and Osama Moselhi

Laser Scanning with Industrial Robot Arm for Raw-wood Fabrication 773

Petras Vestartas and Yves Weinand

Workspace Modeling: Visualization and Pose Estimation of Teleoperated Construction Equipment from

Point Clouds

Jing Dao Chen, Pileun Kim, Dong-Ik Sun, Chang-Soo Han, Yong Han Ahn, Jun Ueda and Yong Cho

A Critical Review of Machine Vision Applications in Construction 789

Saeed Ansari Rad and Mehrdad Arashpour

An Agent-based Approach for Modeling Human-robot Collaboration in Bricklaying 797 Ming-Hui Wu and Jia-Rui Lin

A Vision for Evaluations of Responsive Environments in Future Medical Facilities 805

Daniel Lu, Semiha Ergan, Devin Mann and Katharine Lawrence

Toolbox Spotter: A Computer Vision System for Real World Situational Awareness in Heavy Industries 813

Stuart Eiffert, Alex Wendel, Peter Colborne-Veel, Nicholas Leong, John Gardenier and Nathan Kirchner

Evaluating SLAM 2D and 3D Mappings of Indoor Structures

Yoshihiro Nitta, Derbew Yenet Bogale, Yorimasa Kuba and Zhang Tian

A Novel Audio-Based Machine Learning Model for Automated Detection of Collision Hazards at Construction Sites 829

Khang Dang and Tuyen Le

Training of YOLO Neural Network for the Detection of Fire Emergency Assets 836

Alessandra Corneli, Berardo Naticchia, Massimo Vaccarini , Frederic Bosché and Alessandro Carbonari 
Improvement of 3D Modeling Efficiency and Accuracy of Earthwork Site by Noise Processing Using

Deep Learning and Structure from Motion..... 844

Nobuyoshi Yabuki, Yukako Sakamoto and Tomohiro Fukuda

Real-time Judgment of Workload using Heart Rate and Physical Activity 849

Nobuki Hashiguchi, Lim Yeongjoo, Cyo Sya, Shinichi Kuroishi, Yasuhiro Miyazaki, Shigeo Kitahara, Taizo Kobayashi, Kazuyoshi Tateyama and Kota Kodama

An Integrated Sensor Network Method for Safety Management of Construction Workers 857

Tingsong Chen, Nobuyoshi Yabuki and Tomohiro Fukuda

Data-Driven Worker Detection from Load-View Crane Camera 864

Tanittha Sutjaritvorakul, Axel Vierling and Karsten Berns

Using Deep Learning for Assessment of Workers' Stress and Overload 872

Sahel Eskandar and Saiedeh Razavi

Development of a Workers' Behavior Estimation System Using Sensing Data and Machine Learning Rikuto Tanaka, Nobuyoshi Yabuki and Tomohiro Fukuda

Incident Detection at Construction Sites via Heart-Rate and EMG Signal of Facial Muscle 886

Mizuki Sugimoto, Shunsuke Hamasaki, Ryosuke Yajima, Hiroshi Yamakawa, Kaoru Takakusaki, Keiji Nagatani, Atsushi Yamashita and Hajime Asama

Scenario-Based Construction Safety Training Platform Using Virtual Reality 892

Ankit Gupta and Koshy Varghese

Generation of Orthomosaic Model for Construction Site using Unmanned Aerial Vehicle 900 Alexey Bulgakov, Daher Sayfeddine, Thomas Bock and Awny Fares

Field Application of Tunnel Half Section Inspection System 905

Nobukazu Kamimura, Satoru Nakamura, Daisuke Inoue and Takao Ueno

Challenges in Capturing and Processing UAV based Photographic Data From Construction Sites $\cdots 911$ Saurabh Gupta and Syam Nair

Research and Development on Inspection Technology for Safety Verification of Small-Scale Bridges using Three-Dimensional Model

Kazuhiko Seki, Koichi Iwasa, Satoshi Kubota, Yoshinori Tsukada, Yoshihiro Yasumuro and Ryuichi Imai

Development of Cloud Computing System for Concrete Structure Inspection by Deep Learning Based Infrared Thermography Method

Shogo Hayashi, Koichi Kawanishi, Isao Ujike and Pang-Jo Chun

Stereo Vision based Hazardous Area Detection for Construction Worker's Safety

Doyeop Lee, Numan Khan and Chansik Park 
Artificial Intelligence and Blockchain-based Inspection Data Recording System for Portable Firefighting

Equipment 941

Numan Khan, Doyeop Lee, Ahmed Khairadeen Ali and Chansik Park

Development of Field View Monitor 2 -An assisting function for safety check around a hydraulic excavator using real-time image recognition with monocular cameras948

Yoshihisa Kiyota, Shunsuke Otsuki, Susumu Aizawa and Danting Li

Development of ROV for visual inspection of Concrete Pier Superstructure 954

Toshinari Tanaka, Shuji Nogami, Ema Kato and Tsukasa Kita

An automated Approach to Digitise Railway Bridges 962

Mustafa Al-Adhami, Sagal Rooble, Song Wu, Clara Osuna-Yevenes, Veronica Ruby-Lewis, Mark. Greatrix, Yreilyn Cartagena and Saeed Talebi

Mirror-aided Approach for Surface Flatness Inspection using Laser Scanning 969

Fangxin Li and Min-Koo Kim

A Predictive Model for Scaffolding Man-hours in Heavy Industrial Construction Projects 976

Wenjing Chu, Sanghyeok Han, Zhen Lei, Ulrich Hermann and Di Hu

Ontological Base for Concrete Bridge Rehabilitation Projects 984

Chengke Wu, Rui Jiang, Jun Wang, Jizhuo Huang and Xiangyu Wang

IoT Enabled Framework for Real-time Management of Power-Tools at Construction Projects 992

Ashish Kumar Saxena, Varun Kumar Reja and Koshy Varghese

Web-Based Communication Platform for Decision Making in Early Design Phases 1000

Zhiwei Meng, Ata Zahedi and Frank Petzold

Decision Support System for Site Layout Planning

Abhishek Raj Singh, Ankan Karmakar and Venkata Santosh Kumar Delhi

Factors Affecting the Implementation of Al-based Hearing Protection Technology at Construction Workplace 1014

Yongcan Huang and Tuyen Le

Project Work Breakdown Structure Similarity Estimation Using Semantic and Structural Similarity Measures 1021

Navid Torkanfar and Ehsan Rezazadeh Azar

ABM and GIS Integration for Investigating the Influential Factors Affecting Wildfire Evacuation Performance 1029

Qi Sun and Yelda Turkan

Streamlining Photogrammetry-based 3D Modeling of Construction Sites using a Smartphone, Cloud

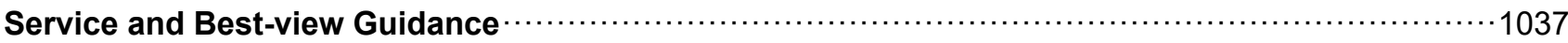

Ryota Moritani, Satoshi Kanai, Kei Akutsu, Kiyotaka Suda, Abdalrahman Elshafey, Nao Urushidate and Mitsuru Nishikawa 
Shigeomi Nishigaki, Katsutoshi Saibara, Takashi Ootsuki and Hirokuni Morikawa

Research on Standardization of Construction Site Time-series Change Information as Learning Data for

Automatic Generation of Work Plan of Construction Machinery in Earthworks

Takashi Otsuki, Hirokuni Morikawa, Yushi Shiiba, Seigo Ogata and Masaharu Moteki

Energy Performance and LCA-driven Computational Design Methodology for Integrating Modular Construction in Adaptation of Concrete Residential Towers in Cold Climates

Sheida Shahi, Patryk Wozniczka, Tristan Truyensb, Ian Trudeau and Carl Haas

A View of Construction Science and Robot Technology Implementation 1069

Hiroshi Yamamoto

Constructible Design for Off-site Prefabricated Structures in Industrial Environments: Review of Mixed Reality Applications

Ankit Shringi, Mehrdad Arashpour and Arnaud Prouzeau

Financial Modeling for Modular and Offsite Construction

Tarek Salama, Gareth Figgess, Mohamed Elsharawy and Hossam Elsokkary

A Novel Methodological Framework of Smart Project Delivery of Modular Integrated Construction 1090 Wei Pan, Mi Pan and Zhenjie Zheng

Block Chain based Remicon Quality Management 1098

Seungwon Cho, Doyeop Lee and Chansik Park

A Conceptual Model for Transformation of Bill of Materials from Offsite Manufacturing to Onsite Construction in Industrialized House-building . 1106

Raafat Hussamadin, Mikael Viklund Tallgren and Gustav Jansson

Study on the Level Concept of Autonomous Construction in Mechanized Construction

Hirokuni Morikawa and Takashi Otsuki

Mask R-CNN Deep Learning-based Approach to Detect Construction Machinery on Jobsites 1122

Hamed Raoofi and Ali Motamedi

Implementation of Unsupervised Learning Method in Rule Learning from Construction Schedules 1128 Boong Yeol Ryoo and Milad Ashtab

Evaluation of Spalling in Bridges Using Machine Vision Method 1136

Eslam Mohammed Abdlekader, Osama Moselhi, Mohamed Marzouk and Tarek Zayed

Improving Construction Project Schedules before Execution 1144

John Fitzsimmons, Ying Hong and Ioannis Brilakis

Automated On-Site Quality Inspection and Reporting Technology for Off-Site Construction (OSC)-based

Seojoon Lee, Soonwook Kwon, Minkyeong Jeong, Syedmobeen Hasan and Alexander Kim 
Hala Nassereddine, Dharmaraj Veeramani and Awad Hanna

Automatic Detection of Air Bubbles with Deep Learning 1168

Takuma Nakabayashi, Koji Wada and Yoshikazu Utsumi

Using a Virtual Reality-based Experiment Environment to Examine Risk Habituation in Construction

\section{Safety}

Namgyun Kim and Changbum Ryan Ahn

Towards a Computational Approach to Quantify Human Experience in Urban Design: A Data Collection Platform

Keundeok Park and Semiha Ergan

Investigation of Changes in Eye-Blink Rate by VR Experiment for Incident Detection at Construction Sites 1191

Shunsuke Hamasaki, Mizuki Sugimoto, Ryosuke Yajima, Atsushi Yamashita, Keiji Nagatani and Hajime Asama

BIM-Aided Scanning Path Planning for Autonomous Surveillance UAVs with LiDAR 1195

Changhao Song, Kai Wang and Jack C. P. Cheng

Research on a Method to Consider Inspection and Processing for Atypical Wood Members Using 3D Laser Scanning

Shunsuke Someya, Yasushi Ikeda, Kensuke Hotta, Seigo Tanaka, Mizuki Hayashi, Mitsuhiro Jokaku and Taito Takahashi

Generative Damage Learning for Concrete Aging Detection using Auto-flight Images 1211

Takato Yasuno, Akira Ishii, Junichiro Fujii, Masazumi Amakata and Yuta Takahashi

Evaluation of Drainage Gradient using Three-dimensional Measurement Data and Physics Engine 1219 Kosei Ishida

Stakeholder Perspectives on the Adoption of Drones in Construction Projects 1227

V. Paul C. Charlesraj and Nijalingamurthy Rakshith

Examination of Efficiency of Bridge Periodic Inspection Using 3D Data (Point Cloud Data and Images) n

Tatsuru Ninomiya, Mami Enomoto, Mitsuharu Shimokawa, Tatsuya Hattori and Yasushi Nitta

Experimental Result of Third-person View Generation using Deliberately Delayed Omni-directional Video

Akira Sakata, Yasushi Hada, Rei Hojo, Masahiro Munemoto, Yoshito Takeshita, Tsubasa Asuma and Shigeo Kitahara

Construction Operation Assessment and Correction Using Laser Scanning and Projection Feedback Alexei Pevzner, Saed Hasan, Rafael Sacks and Amir Degani 
Jia-Rui Lin

Five-dimensional Simulation of Bridge Engineering Based on BIM and VR

Kun-Chi Wang, Sheng-Han Tung, Wei-Chih Chen and Zi-Chi Zhao

Digital Twinning of Railway Overhead Line Equipment from Airborne LiDAR Data

M. R. Mahendrini Fernando Ariyachandra and Ioannis Brilakis

A Shared Ontology for Logistics Information Management in the Construction Industry

Yuan Zheng, Müge Tetik, Seppo Törmä, Antti Peltokorpi and Olli Seppänen

Visualization of the Progress Management of Earthwork Volume at Construction Jobsite 1286

Hajime Honda, Akifumi Minami, Yoshihiko Takahashi, Seishi Tajima, Takashi Ohtsuki and Yushi Shiiba

Incentivizing High-Quality Data Sets in Construction Using Blockchain: A Feasibility Study in the Swiss Industry

Jens J. Hunhevicz, Tobias Schraner and Daniel M. Hall

A Holistic Framework for the Implementation of Big Data throughout a Construction Project Lifecycle

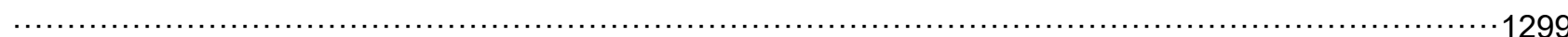
Makram Bou Hatoum, Melanie Piskernik and Hala Nassereddine

Automatic Analysis of Idling in Excavator's Operations Based on Excavator-Truck Relationships $* 1307$ Chen Chen, Zhenhua Zhu and Amin Hammad

Construction 4.0: A Roadmap to Shaping the Future of Construction 1314

Mahmoud El Jazzar, Harald Urban, Christian Schranz, and Hala Nassereddine

Deep Learning-based Question Answering System for Proactive Disaster Management 1322

Yohan Kim, Jiu Sohn, Seongdeok Bang and Hyoungkwan Kim

Overall Utilization of Information and Communication Technologies in Excavation Work and

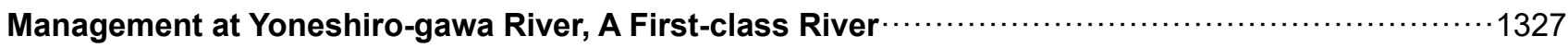

Tatsuro Masu, Akihiro Ishii, Fumihiro Tamori, Hanako Hatakeyama, Yutaka Suzuki, Satoshi Shirato and Yurie Abe

Weakly Supervised Defect Detection using Acoustic Data based on Positive and Negative Constraints 1331

Jun Younes Louhi Kasahara, Atsushi Yamashita and Hajime Asama

Road Maintenance Management System Using 3D Data by Terrestrial Laser Scanner and UAV $\cdots \cdots 1337$ Satoshi Kubota, Ryosuke Hata, Kotaro Nishi, Chiyuan Ho and Yoshihiro Yasumuro

Traffic Regulation Technology by Movable Barriers 1344 Toshiharu Tanikawa and Tohya Okishio

Development and Verification of Inspection Method for Concrete Surface utilizing Digital Camera 1351 Shungo Matsui, Yoshimasa Nakata, Hidenori Shitashimizu, Ryota Nakatsuji, Takeshi Ueda and Naoiki Maehara 
MLIT's Initiatives for Promotion the Efficient Construction and Inspection by using new Technologies such as Al and Robots in Japan.

Kenichi Watanabe

Track Similarity-based Typhoon Search Engine for Disaster Preparedness

Chun-Mo Hsieh, Cheng-Yu Ho, Hung-Kai Kung, Hao-Yung Chan, Meng-Han Tsai and Yun-Cheng Tsai

Cracks Detection using Artificial Intelligence to Enhance Inspection Efficiency and Analyze the Critical

Defects

Fawaz Habbal, Abdualla Alnuaimi, Mohammed Al Shamsi, Saleh Alshaibah and Thuraya Aldarmaki Thuraya.Aldarmaki

Smart Tunnel Inspection and Assessment using Mobile Inspection Vehicle, Non-Contact Radar and Al 1373

Toru Yasuda, Hideki Yamamoto, Mami Enomoto and Yasushi Nitta

Applications of Building Information Modeling (BIM) in Disaster Resilience: Present Status and Future Trends 1380

Sadegh Khanmohammadi, Mehrdad Arashpour and Yu Bai

Integrating BIM- and Cost-included Information Container with Blockchain for Construction Automated Payment using Billing Model and Smart Contracts 1388

Xuling Ye, Katharina Sigalov and Markus König

An Agent-based Framework for Evaluating Location-based Risk Score in Indoor Emergency Evacuation 1396

Tianlun Cai, Jiamou Liu, Hong Zheng, Yupan Wang and Vicente Gonzalez

A Framework for Camera Planning in Construction Site using 4D BIM and VPL

Si Tran, Ahmed Khairadeen Ali, Numan Khan, Doyeop Lee and Chansik Park

Safe and Lean Location-based Construction Scheduling 1409

Beidi Li, Carl Schultz, Jürgen Melzner, Olga Golovina, and Jochen Teizer

Don't Risk Your Real Estate Actions to Realize Efficient Project Risk Management using the BIM Method Maike Eilers, Carla Pütz, Manfred Helmus and Anica Meins-Becker

Introduction of the New Safety Concept "Safety2.0" to Reduce the Risk of Machinery Accidents $\cdots 1424$ Hidesato Kojima, Takaya Fujii, Yasushi Mihara and Hiroaki Ihara

Applying ANN to the Al Utilization in Forecasting Planning Risks in Construction 1431

Fawaz Habbal, Firas Habbal, Abdualla Alnuaimi, Anwar Alshimmari, Nawal Alhanaee and Ammar Safi

Development of A Mobile Robot pulling An Omni-directional Cart for A Construction Site 1438

Yusuke Takahashi, Yoshiro Hada and Satoru Nakamura

Taufik Akbar Sitompul, Simon Roysson and José Rosa 
Kazuki Sumi

Preliminary Development of a Powerful and Backdrivable Robot Gripper Using Magnetorheological

Fluid

Sahil Shembekar, Mitsuhiro Kamezaki, Peizhi Zhang, Zhouyi He, Tsunoda Ryuichiro, Hiroyuki Sakamoto and Shigeki Sugano

Development and Application of a Fire Resistive Covering Spraying Robot to Building Construction Site Yuichi Ikeda, Hirofumi Segawa and Nobuyoshi Yabuki

A Cable Driven Parallel Robot with a Modular End Effector for the Installation of Curtain Wall Modules

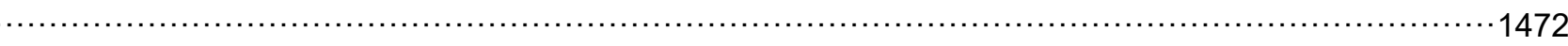

Kepa Iturralde, Malte Feucht, Rongbo Hu, Wen Pan, Marcel Schlandt, Thomas Linner, Thomas Bock, JeanBaptiste Izard, Ibon Eskudero, Mariola Rodriguez, Jose Gorrotxategi, Julen Astudillo, Joao Cavalcanti, Marc Gouttefarde, Marc Fabritius, Christoph Martin, Tomas Henninge, Stein M. Nornes, Yngve Jacobsen, A. Pracucci, Jesús Cañada, José David Jimenez-Vicaria, Carlo Paulotto, Ruben Alonso and Lorenzo Elia

Bi-Directional Communication Bridge for State Synchronization between Digital Twin Simulations and Physical Construction Robots 1480

Ci-Jyun Liang, Wes McGee, Carol Menassa and Vineet Kamat

Parallel Kinematic Construction Robot for AEC Industry 1488

Maike Klöckner, Mathias Haage, Klas Nilsson, Anders Robertsson and Ronny Andersson

Design-to-Robotic-Production and -Assembly for Architectural Hybrid Structures 1496

Henriette Bier, Arwin Hidding and Marco Galli

Design and Synthesis of the Localization System for the On-site Construction Robot 1501 Wen Pan, Rui Li and Thomas Bock

Online Synchronization of Building Model for On-Site Mobile Robotic Construction 1508 Selen Ercan Jenny, Hermann Blum, Abel Gawel, Roland Siegwart, Fabio Gramazio and Matthias Kohler

A Methodology to Monitor Construction Progress Using Autonomous Robots 1515 Samuel A. Prieto, Borja Garcia de Soto and Antonio Adan

Digital Twin Technology Utilizing Robots and Deep Learning 1523 Fuminori Yamasaki

Real-Time Process-Level Digital Twin for Collaborative Human-Robot Construction Work 1528 Xi Wang, Ci-Jyun Liang, Carol Menassa and Vineet Kamat

Research and Development of Construction Technology in Social Cooperation Program "Intelligent Construction System" 1536

Shota Chikushi, Jun Younes Louhi Kasahara, Hiromitsu Fujii, Yusuke Tamura, Angela Faragasso, Hiroshi Yamakawa, Keiji Nagatani, Yonghoon Ji, Shinya Aoki, Takumi Chiba, Shingo Yamamoto, Kazuhiro Chayama, Atsushi Yamashita and Hajime Asama 
Automated Framework for the Optimisation of Spatial Layouts for Concrete Structures Reinforced with

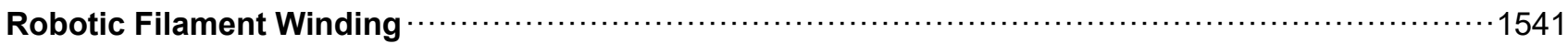

Robin Oval, Eduardo Costa, Diana Thomas-Mcewen, Saverio Spadea, John Orr and Paul Shepherd

Adopting Off-site Manufacturing, and Automation and Robotics Technologies in Energy-efficient

Building 1549

Wen Pan, Kepa Iturralde Lerchundi, Rongbo Hu, Thomas Linner and Thomas Bock

Analysis on the Implementation Mechanism of an Inspection Robot for Glass Curtain Walls in High-rise Buildings 1556

Shiyao Cai, Zhiliang Ma and Jianfeng Guo

Application of Robots to the Construction of Complex Structures using Standardized Timbers $\cdots \cdots 1562$ Yi Leng , Xingyu Shi and Fukuda Hiroatsu

A Preliminary Comparison Between Manual and Robotic Construction of Wooden Structure Architecture 1568

Lu Wang, Hiroatsu Fukuda and Xinyu Shi

Towards 3D Perception and Closed-Loop Control for 3D Construction Printing 1576 Xuchu Xu, Ruoyu Wang, Qiming Cao and Chen Feng

Robotics Autonomous Surveillance Algorithms for Assessing Construction Automation and Completion Progress 1584

Firas Habbal, Abdualla AlNuaimi, Dhoha Alhmoudi, Mariam Alrayssi and Ahmed Alali

Oscillation Reduction for Knuckle Cranes 1590

Michele Ambrosino, Arnaud Dawans, Brent Thierens and Emanuele Garone

Bridge Inspection with Aerial Robots and Computer Vision: A Japanese National Initiative 1598 Jacob J. Lin, Amir Ibrahim, Shubham Sarwade, Mani Golparvar-Fard, Yasushi Nitta, Hirokuni Moirkawa and Yoshihiko Fukuchi 